\title{
Cryo-FIB: Overcoming the Hurdle of Sample Preparation for In Situ Cryo-Electron Tomography
}

\author{
Miroslava Schaffer
}

Max Planck Institute of Biochemistry, Dep. of Molecular Structural Biology, Martinsried, Germany

Sample preparation is the first, and often most crucial and limiting, step in transmission electron microcopy (TEM) investigation. While cryo-TEM can provide images of incredible detail and resolution from frozen-hydrated specimens, the resolution declines with increasing sample thickness. Useful data can only be obtained from materials up to a thickness of a few hundred nanometers, a limit easily exceeded by many cells and tissues. To study biological macromolecules within their native environment, it is thus necessary to thin most cellular samples prior the TEM investigation.

While the final thickness and quality of the prepared sample has a strong impact on the attainable data quality, it is also absolute key to prevent damage or alteration of the volume of interest during the preparation process. Over the last decade, cryo-focused ion beam (cryo-FIB) microscopy has been shown to be a suitable tool for such a task [1,2]. The development of a reliable lamella preparation technique from plunge-frozen specimens has opened the door to novel and exciting research. With cryoFIB as a new and crucial 'enabling' technique, we can now study biological macromolecules in their various structural states observed directly within their natural environment [3-7].

In the standard cryo-FIB lamella preparation workflow [8,9], the plunge-frozen specimen is milled under a shallow angle with respect to the grid surface. Sample material is removed by ion-beam sputtering both above and below the feature of interest, such that the remaining material forms a lamella of $200 \mathrm{~nm}$ thickness or less over an area of several $\mu \mathrm{m}^{2}$. While the geometry is simple and the preparation of multiple lamellas on a single sample grid can be performed within a few work hours, the final quality of the lamella is one of the main factors determining failure or success of the following cryo-TEM investigation. A lamella needs to be homogenously thin over the area. The beam-sensitive biological material must not have been damaged by ion or electron irradiation, and there must be minimal ice contamination on both lamella surfaces. Further technique developments are focused on optimizations for special applications such as correlative microscopy, lamella surface coatings for Volta phase plates imaging, and the adjustment of milling parameters and routines for different specimens.

\section{References:}

[1] M Marko et al, Nat Methods 4 (2007), p. 215.

[2] A Rigort et al, PNAS 109 (2012), p. 4449.

[3] B Engel et al, eLife 4 (2015), e04889

[4] S Albert et al, PNAS 114 (2017), p. 13726.

[5] YS Bykov et al, eLife 6 (2017), e32493.

[6] ES Freeman Rosenzweig et al, Cell 171 (2017), p. 148.

[7] S Pfeffer et al, Nat Commun 8 (2017), p. 14516.

[8] M Schaffer et al, bio-protocol 5 (2015), e1575.

[9] M Schaffer et al, J Struct Biol. 197 (2017), p. 73. 

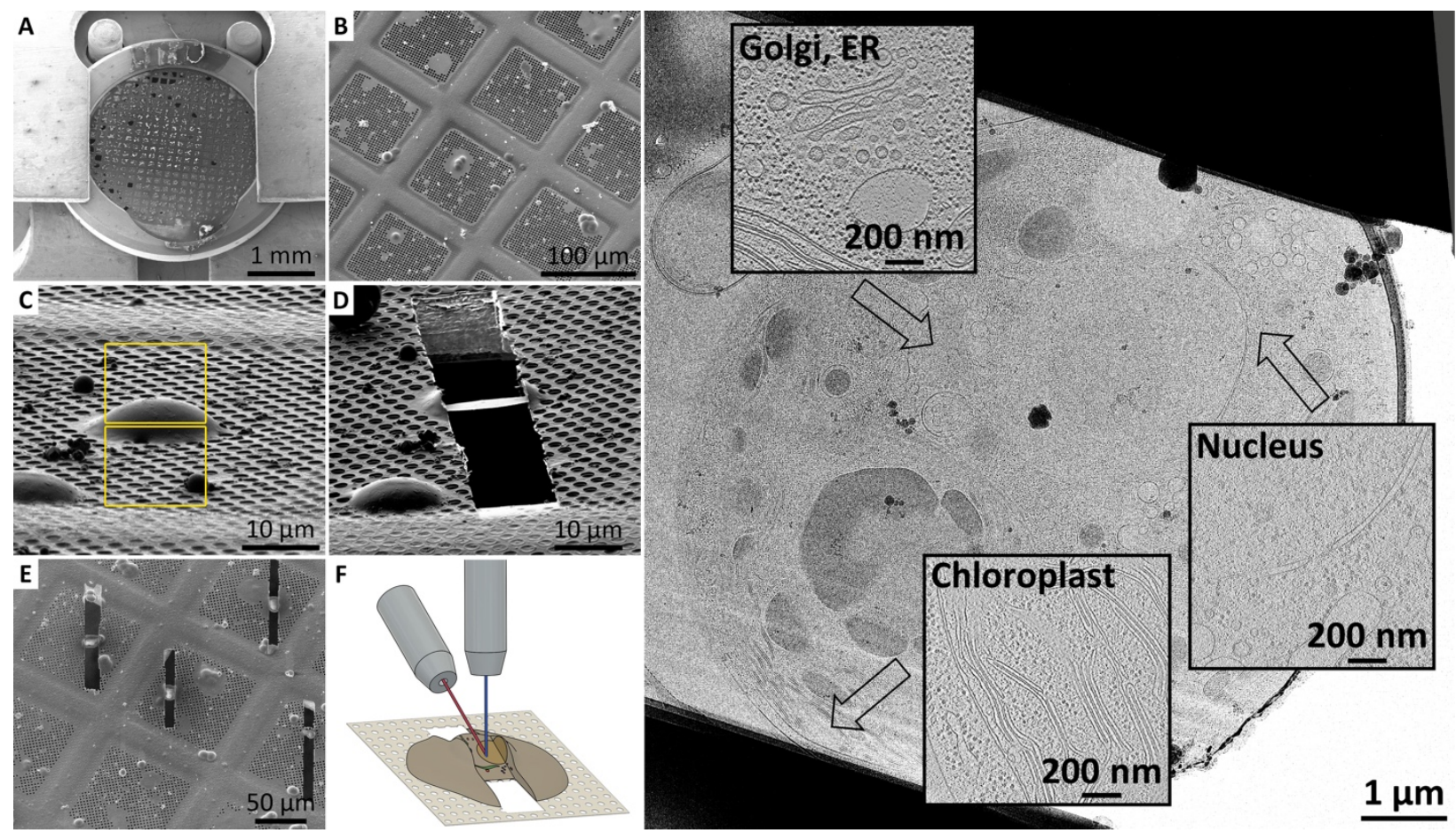

Figure 1. Cryo-FIB lamella preparation of a plunge-frozen specimen. (A,B) SEM images of the grid on the FIB cryo-stage. (C,D) FIB-induced secondary electron image of the grid with frozen Chlamydomonas reinhardtii cells before and after the milling. Two rectangle patterns indicate the areas where the material is cut. (E) SEM image of the grid after preparing multiple lamellas. (F) Schematic of a finished lamella. The direction of ion beam and the electron beam are shown as red and blue rays, respectively. (G) Cryo-EM/ET images of the final lamella. Different organelles of the Chlamydomonas cell are highlighted by arrows, and slices from tomographic volumes depicting these organelles are shown in the insets. 